\title{
DETECTION OF METALLO- $\beta$-LACTAMASE PRODUCTION IN GRAM-NEGATIVE BACILLI ISOLATED FROM PATIENTS ATTENDING A TERTIARY CARE HOSPITAL IN BIHAR
}

\author{
Nishant Upadhyay1, Sangeeta Dey², Priyanka Paul Biswas³, Dhananjay Kumar ${ }^{4}$,Krishan Nandan ${ }^{5}$, Aninda Sen ${ }^{6}$, Dharmendra Singh 7 , \\ Mahadeo Mandal ${ }^{8}$ \\ 1 Postgraduate Student, Department of Microbiology, Katihar Medical College and Hospital, Katihar, Bihar. \\ 2 Professor and HOD, Department of Microbiology, Katihar Medical College and Hospital, Katihar, Bihar. \\ ${ }^{3}$ Assistant Professor, Department of Microbiology, Katihar Medical College and Hospital, Katihar, Bihar. \\ ${ }^{4}$ Assistant Professor, Department of Microbiology, Katihar Medical College and Hospital, Katihar, Bihar. \\ ${ }^{5}$ Associate Professor, Department of Microbiology, Katihar Medical College and Hospital, Katihar, Bihar. \\ ${ }^{6}$ Professor, Department of Microbiology, Katihar Medical College and Hospital, Katihar, Bihar. \\ ${ }^{7}$ Tutor, Department of Microbiology, Katihar Medical College and Hospital, Katihar, Bihar. \\ ${ }^{8}$ Postgraduate Student, Department of Microbiology, Katihar Medical College and Hospital, Katihar, Bihar.
}

\section{ABSTRACT}

\section{BACKGROUND}

MBL (Metallo-beta-lactamase) production by GNB (Gram-negative bacilli) has recently emerged as one of the most worrisome resistance mechanisms. MBL producing GNB have been reported from different parts of the world. There is no effective MBL inhibitor developed to combat them in vivo, although in vitro metal chelators like EDTA can help augment their detection by phenotypic methods. Carbapenems are the last resort in treating infections caused by GNB, occurrence of MBL genes in these isolates leaves us with few therapeutic options like polymyxins which are potentially toxic compounds.

The aim of this study was to detect the production of MBL in GNB obtained from patients attending Katihar Medical College and Hospital in Katihar district of Bihar.

\section{MATERIALS AND METHODS}

GNB were identified as per standard protocol and were tested against different antimicrobial agents on MHA (Mueller-Hinton Agar) by modified Kirby-Bauer Disc Diffusion Technique. Carbapenemase production was detected by MHT (Modified Hodge Test). MBL detection was done with DDST (Double Disk Synergy Test) and DPT (Disk Potentiation Test) in all 103 GNB isolates included in our study.

\section{RESULTS}

Out of a total of 103 GNB isolated 18 were detected as MBL producers. Maximum MBL production was seen in P. aeruginosa isolates 13/22 (72.22\%) followed by E. coli 3/51 (16.67\%) and K. pneumoniae 2/19 (11.11\%). All the three methods for detection of MBL were found equally useful.

\section{CONCLUSION}

MBL production was found to be fairly high in our region, especially in Pseudomonas aeruginosa, in which 59.09\% were MBL producers. These findings are a cause for much alarm, as this college is situated in a rural area catering mainly to rural patients, as regards to the isolation of such a high number of MBL producing isolates. All three phenotypic tests performed equally. Out of the three methods tested, the DPT was the easiest to perform. It would be wise to perform any one of these tests routinely in diagnostic laboratories especially on strains showing resistance to any one of the carbapenem antibiotics.

\section{KEYWORDS}

Gram-negative Bacilli, MBLs.

HOW TO CITE THIS ARTICLE: Upadhyay N, Dey S, Biswas PP, et al. Detection of metallo- $\beta$-lactamase production in Gram-negative bacilli isolated from patients attending a tertiary care hospital in Bihar. J. Evolution Med. Dent. Sci. 2017;6(26):2130-2133, DOI: $10.14260 /$ Jemds/2017/462

\begin{tabular}{|c|c|}
\hline $\begin{array}{l}\text { BACKGROUND } \\
\text { MBLs are enzymes that have the ability to hydrolyse } \beta \text {-lactam } \\
\text { antibiotics which require zinc at active site. MBLs have } \\
\text { recently emerged as one the most worrisome resistance } \\
\text { mechanisms as MBL genes are located on integron structures }\end{array}$ & $\begin{array}{l}\text { that reside on mobile genetic elements such as plasmids or } \\
\text { transposons, which gives them ability for widespread } \\
\text { dissemination. }{ }^{1} \\
\text { Acquisition of MBL gene invariably mediates broad } \\
\text { spectrum } \beta \text {-lactam resistance in Pseudomonas aeruginosa, }\end{array}$ \\
\hline $\begin{array}{l}\text { Financial or Other, Competing Interest: None. } \\
\text { Submission 23-02-2017, Peer Review 19-03-2017, } \\
\text { Acceptance 25-03-2017, Published 30-03-2017. } \\
\text { Corresponding Author: } \\
\text { Dr. Nishant Upadhyay, } \\
\text { S/o. Dr. C. M. Upadhyay, } \\
\text { M.G. Road, Near Panchwati Hotel, } \\
\text { Bhagalpur-812001, Bihar. } \\
\text { E-mail: drnishantupadhyaymicro@gmail.com } \\
\text { DOI: } 10.14260 / \text { jemds/2017/462 } \\
\text { (c) } \$(\Theta)\end{array}$ & $\begin{array}{l}\text { but the level of in vitro resistance in Acinetobacter sp. an } \\
\text { Enterobacteriaceae is less dependable. Their clinic } \\
\text { significance is further embellished by their ability } \\
\text { hydrolyse all } \beta \text {-lactams and by the fact that currently there } \\
\text { no clinical inhibitor, nor is there likely to be for th } \\
\text { foreseeable future. } \\
\text { The first indication of mobile MBLs was with th } \\
\text { discovery of Pseudomonas aeruginosa strain GN17203 } \\
\text { Japan in I988.2 }\end{array}$ \\
\hline
\end{tabular}


The belief that mobile MBLs genes were solely a distant Japanese problem was negated with the advent of bla-IMP-2 in 1997 and bla-IMP-5 in 1998 from Italy and Portugal, respectively. ${ }^{2}$

VIM was described first in Verona, Italy, from a Pseudomonas aeruginosa isolate. A clinical Pseudomonas aeruginosa isolate from 1997 from Sao Paulo, Brazil, was analysed as part of the SENTRY surveillance program and shown to contain a novel gene, designated bla-SPM-1 (Sao Paulo MBL). ${ }^{2}$

In 2002, five Pseudomonas aeruginosa isolates were recovered from different patients from a medical site in Dusseldorf, Germany and shown to possess a novel class-B $\beta$ lactamase designated GIM-1 (German imipenemase). ${ }^{2}$

New Delhi metallo-beta-lactamase-1 (NDM-1) has been reported from many countries and its origin has been traced to Asia. This MBL is plasmid borne and has propensity to spread between species through horizontal transfer.

The isolates produce zinc dependent metallo-betalactamases which hydrolyse beta-lactam ring of the antibiotics and render them inactive. They require zinc for their activity. The detection of MBL has become very important in all laboratories to determine the ultimate clinical outcome in all those patients who are infected with MBL producing strains. Even smaller laboratories must try and identify these MBL strains. Their wide spectrum of activity leaves us with few therapeutic options with no effective MBL inhibitor developed to combat them in vivo, although in vitro metal chelators like EDTA can help augment their detection by phenotypic methods. Clinical and Laboratory Standards Institute 4 has not laid down any specific guidelines though there are several screening methods for detection and confirmation of MBL production in GNB. Currently the most accepted method is MBL E-Test. However, due to high cost many laboratories use alternative methods as the DDST and DPT.2

\section{MATERIALS AND METHODS}

Patients of both sexes and all age groups were included in this study after obtaining Institutional Ethical Committee clearance and informed consent from each and every patient. All samples were subjected to microscopic examination and cultured on standard laboratory media. GNB were identified as per standard protocol. ${ }^{3}$ Antibiotic susceptibility tests were put up by modified Kirby-Bauer's disc diffusion method ${ }^{4}$ using a panel of antibiotics disks obtained from HiMedia (Mumbai).

\section{MBL Screening}

Screening for MBL production was done in all isolates by MHT, DDST and DPT.

\section{Modified Hodge Test ${ }^{5}$}

A suspension of 0.5 McFarland standard of the indicator organism (E. coli ATCC 25922) was prepared. It was inoculated on the surface of a MHA plate. The test strain was heavily streaked from the centre to periphery of the plate. The plate was allowed to stand for 15 minutes. An imipenem $10 \mu \mathrm{g}$ disk was placed in the centre. $10 \mu \mathrm{L}$ of $50 \mathrm{mM}$ zinc sulphate $(140 \mu \mathrm{g})$ was added to the imipenem disks. The plates were incubated overnight at $37^{\circ} \mathrm{C}$. After overnight incubation the plates were observed for the presence of "clover leaf" shaped zone of inhibition. The plates with such zones were interpreted as Modified Hodge test positive.

\section{Disc Potentiation Test 6}

A 0.5 McFarland standard suspension from log phase growth of the test organism was prepared in Mueller Hinton broth or normal saline. This suspension was inoculated onto the plates of MHA. Two $10 \mu \mathrm{g}$ imipenem disks were placed on the plate and $5 \mu \mathrm{L}$ EDTA $(750 \mu \mathrm{g})$ was added to one of the disks. The plates were incubated at $37{ }^{\circ} \mathrm{C}$ for 18 hours.

(Solution of $0.5 \mathrm{M}$ EDTA was prepared by dissolving $186.1 \mathrm{~g}$ of disodium EDTA. $2 \mathrm{H}_{2} \mathrm{O}$ in $1000 \mathrm{~mL}$ distilled water and $\mathrm{pH}$ was adjusted to 8.0 by using $\mathrm{NaOH}$. The mixture was sterilized by autoclaving)

\section{Reading}

The inhibition zones of the imipenem and imipenem-EDTA disks were measured with the help of a scale. An increase in the zone diameter $\geq 7 \mathrm{~mm}$ in presence of EDTA indicated the presence of MBL. Figure 1.

\section{Double Disk Synergy Test ${ }^{7}$}

A suspension equivalent to 0.5 McFarland standards from a log phase of the test organism was prepared in MuellerHinton broth. This was inoculated on MHA plate and $30 \mu \mathrm{g}$ ceftazidime disk and $10 \mu \mathrm{g}$ imipenem disk was applied on the plates. The distance between the disks was kept at 4 to $5 \mathrm{~cm}$. A blank filter paper disk was placed near the ceftazidime and imipenem disk with a centre to centre distance of 1.0 to 2.5 cm. $5 \mu \mathrm{L}$ of EDTA was added to the filter paper disk and plates incubated at $37^{\circ} \mathrm{C}$ overnight. After overnight incubation, even a small synergistic zone of inhibition was interpreted as positive. Figure 2 .

\section{RESULTS AND OBSERVATIONS}

Out of a total of 3056 samples received in the laboratory from January 2015 to June 2016, 668 samples showed growth of different bacterial isolates. 223/668 (33.38\%) showed growth of GPC in pure culture, $342 / 668$ (51.20\%) showed mixed growth and 103/668 (15.42\%) showed growth of GNB in pure form which were processed for further study. Maximum number of cases was seen in the age group of 21 to 30 years $28 / 103(27.18 \%)$ followed by the age group of 31 to 40 years 25/103 (24.27). In females, maximum number of GNB was isolated from age group 21-30 years 22/28 $(78.57 \%)$ whereas in males, maximum number of GNB were isolated from age group 0-10 years 14/21 (66.67\%). The overall male to female ratio was 1:1.15.

Majority of cases with GNB infections were from Obstetrics and Gynaecology department 35.92\% (37/103) followed by Surgery $22.33 \%$ (23/103), Paediatrics $20.38 \%$ (21/103) and Medicine 19.42\% (20/103).

Meropenem resistance was seen in 72/103(69.90\%) of GNB isolates and 27/103(22.33\%) isolates were found to be imipenem resistant.

The detection rates of carbapenemases production by three different phenotypic method namely MHT, DPT and DDST was $17.47 \%$ (18/103). All the three phenotypic methods of MBL detection showed $100 \%$ correlation. Table 1 .

Maximum production of MBL was seen in Pseudomonas aeruginosa isolates $13 / 18(72.22 \%)$. This was followed by E. coli 3/18 (16.67\%) and Klebsiella pneumonia 2/18 (11.11\%). Other Gram-negative isolates did not show the presence of MBLs. Table 2. 
Majority of MBL positive strains $88.88 \%$ (16/18) were isolated from urine. None of the MBL producing isolates were from the out-patient department. Maximum numbers of MBL positive isolates were from Surgery in-patients $62.50 \%$ followed by Obstetrics and Gynaecology in-patients $50.00 \%$.

GNB (other than Pseudomonas aeruginosa) showed maximum resistance seen to cefuroxime 73/81 (90.13\%) followed by cefotaxime $71 / 81$ (87.65\%), ertapenem $58 / 81$ (71.60\%), meropenem 56/81 (69.14\%). 18.82\% of strains were resistant to imipenem. No resistance was seen with Colistin which showed $100.00 \%$ sensitivity.

Pseudomonas aeruginosa isolates showed $100.00 \%$ resistance to cefotaxime, $68.19 \%$ of strains showed resistance to amikacin, meropenem and netilmicin, followed by levofloxacin, gentamicin and ceftazidime 59.09\% each. Resistance to imipenem was seen in $54.55 \%$ and no resistance was seen with Colistin which showed $100.00 \%$ sensitivity.

Resistance to amikacin, cefotaxime, ertapenem, meropenem, cefuroxime, levofloxacin, netilmicin, gentamicin and ceftazidime was $100.00 \%$ among MBL producers, followed by imipenem $83.33 \%$ and nitrofurantoin $60.00 \%$ which was tested against urinary isolates only. In MBL negative cases, the maximum resistance seen with cefotaxime $88.23 \%$ and cefuroxime $78.82 \%$ followed by meropenem $63.52 \%$, levofloxacin $55.29 \%$, netilmicin $28.57 \%$, gentamicin $23.52 \%$ and amikacin $15.29 \%$. Table 3.

\begin{tabular}{|c|c|c|}
\hline Name of Test & Positive (\%) & Negative (\%) \\
\hline Modified Hodge Test & $18(17.47)$ & $85(82.52)$ \\
\hline DPT & $18(17.47)$ & $85(82.52)$ \\
\hline DDST & $18(17.47)$ & $85(82.52)$ \\
\hline \multicolumn{3}{|c|}{ Table 1. Carbapenemase Production by Gram-negative } \\
bacilli \\
\hline
\end{tabular}

\begin{tabular}{|c|c|c|c|}
\hline GNB & MBL +ve (\%) & MBL -ve (\%) & TOTAL \\
\hline Escherichia coli & $3(16.67)$ & $48(56.47)$ & $51(49.51)$ \\
\hline K. pneumoniae & $2(11.11)$ & $17(20.00)$ & $19(18.44)$ \\
\hline P. aeruginosa & $13(72.22)$ & $9(10.58)$ & $22(21.35)$ \\
\hline C. freundii & 0.00 & $4(4.70)$ & $4(3.88)$ \\
\hline E. cloacae & 0.00 & $1(1.17)$ & $1(0.97)$ \\
\hline P. mirabilis & 0.00 & $5(5.88)$ & $5(4.85)$ \\
\hline A. baumannii & 0.00 & $1(1.17)$ & $1(0.97)$ \\
\hline Total & 18 & 85 & 103 \\
\hline
\end{tabular}

\begin{tabular}{|c|c|c|c|c|}
\hline \multirow{2}{*}{ Antibiotic } & \multicolumn{2}{|c|}{ MBL Positive n= 18 } & MBL Negative n=85 \\
\cline { 2 - 5 } & No. & $\mathbf{\%}$ & No. & \% \\
\hline Amikacin & 18 & 100.00 & 13 & 15.29 \\
\hline Cefotaxime & 18 & 100.00 & 75 & 88.23 \\
\hline Meropenem & 18 & 100.00 & 54 & 63.52 \\
\hline Cefuroxime $\ddagger$ & 05 & 100.00 & 67 & 78.82 \\
\hline Imipenem & 15 & 83.33 & 16 & 18.82 \\
\hline Levofloxacin & 18 & 100.00 & 47 & 55.29 \\
\hline Colistin & 00 & 00.00 & 00 & 00.00 \\
\hline Nitrofurantoin* & 03 & 60.00 & 28 & 41.79 \\
\hline Netilmicin† $\dagger$ & 13 & 100.00 & 04 & 28.57 \\
\hline Gentamicin & 18 & 100.00 & 20 & 23.52 \\
\hline Ceftazidime $\dagger$ & 13 & 100.00 & 00 & 00.00 \\
\hline Table 3. Resistance Pattern of MBL-positive and MBL- \\
negative Isolates \\
\hline
\end{tabular}

*Only for Urinary Isolates of GNB, $\uparrow$ Only for P. Aeruginosa, ‡ Used only for GNB other than Pseudomonas

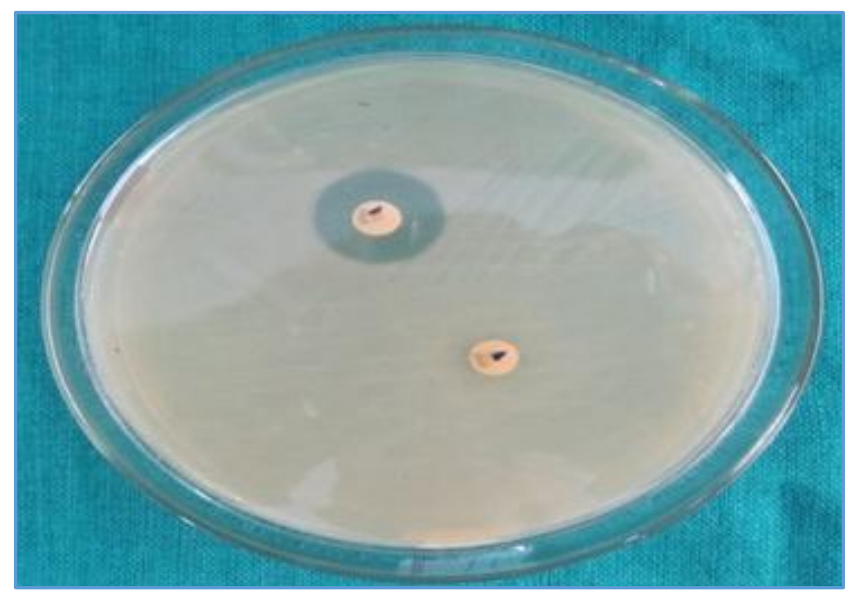

Figure 1. Showing Disk Potentiation Test of an MBL-Positive GNB Isolate

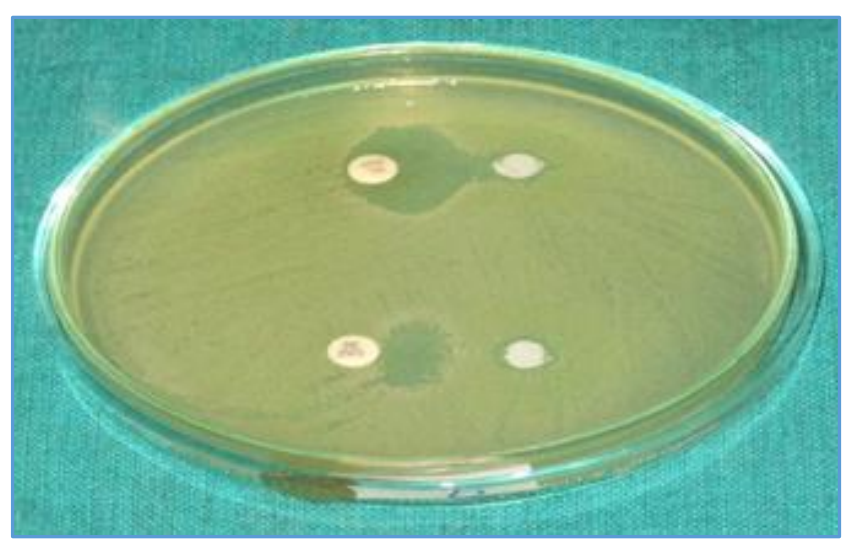

Figure 2. Showing Double Disk Synergy Test in case of MBL-Positive GNB Isolate

\section{DISCUSSION}

Maximum MBL production was seen in Pseudomonas aeruginosa isolates $72.22 \%$ followed by Escherichia coli $16.67 \%$ and Klebsiella pneumonia $11.11 \%$. MBL production was not seen with other isolates viz. Citrobacter freundii, Enterobacter cloacae, Proteus mirabilis and Acinetobacter baumannii. Out of a total of 22 Pseudomonas aeruginosa isolates, 13 were MBL producers, $59.09 \%$ as compared to 8.05\% reported by other authors. 8 This study; however, was conducted 8 years back and probably indicates an increasing trend in isolation of MBL producers. As far as Enterobacteriaceae is concerned out of a total $70 \mathrm{E}$. coli and K. pneumoniae isolates, only $7.14 \%$ (5/70) were found to be MBL producers. Few other authors on the other hand have reported that $25.53 \%$ of their Enterobacteriaceae isolates were MBL producers which is fairly high compared to the present study. ${ }^{9}$

In the present study, three different types of phenotypic tests were done for detection of carbapenemases viz. the MHT, DPT and DDST. All these tests showed 100\% correlation with each other. However, as far as ease of performance of each of these tests is concerned the DPT was the easiest to perform. Other authors also emphasised that Imipenem-EDTA Combined Disk Test can be used as a convenient and cost effective MBL screening method in clinical Microbiology laboratory. ${ }^{10}$

A very high degree of resistance was seen with MBL positive strains. All $18 \mathrm{MBL}$ positive showed $100 \%$ resistance 
to amikacin, cefotaxime, meropenem, levofloxacin and gentamicin. In addition to this, all MBL positive Pseudomonas aeruginosa isolates were resistant to netilmicin and ceftazidime. Colistin was the only antibiotic to which all MBL producers were sensitive.

Other authors have also reported $100 \%$ resistance to gentamicin, co-trimoxazole, cefotaxime, ceftazidime in their E. coli isolates, which is similar to this study. ${ }^{11}$

Other studies on NDM-1 producing enteric isolates reported that $61.64 \%, 69.23 \%$ and $76.92 \%$ of their isolates were resistant to amikacin, tobramycin and gentamicin respectively. ${ }^{12}$

\section{CONCLUSION}

MBL production was found to be fairly high in our region, especially in Pseudomonas aeruginosa in which 59.09\% were MBL producers. MBL production was also relatively high in Klebsiella pneumoniae isolates. These findings are a cause for much alarm, as this college is situated in a rural area catering mainly to rural patients, as regards to the isolation of such a high number of MBL producing isolates. This poses a genuine threat, as far as patient care is concerned, as majority of these MBL were found to be resistant to almost all the antibiotics against which they were tested except Colistin.

As far as detection of MBLs by phenotypic methods is concerned, in our study all three phenotypic tests performed equally. Out of the three methods tested, the DPT was the easiest to perform and has the potential to be incorporated in even small diagnostic laboratories without any constraints on material, infrastructure and manpower. It would be wise to perform any one of these tests routinely in diagnostic laboratories especially on strains showing resistance to any one of the carbapenem antibiotics.

\section{REFERENCES}

[1] Bush K, Jacoby GA. Updated functional classification of beta-lactamases. Antimicrob Agents Chemother 2010;54(3):969-76.

[2] Walsh TR, Toleman MA, Poirel L, et al. Metallo-betalactamases: the quiet before the storm? Clin Microbiol Rev 2005;18(2):306-25.

[3] Collee JG, Miles RS, Watt B, et al. Tests for identification of bacteria. In: Collee JG, Fraser AG, Marmion BP, (eds). Mackie and McCartney practical medical microbiology. 14th edn. Churchill Livingstone: New Delhi 2006:131-49.
[4] Performance standards for antimicrobial susceptibility testing: twenty-fifth informational supplement, M100-S25. Clinical and Laboratory Standards Institute 2013;35(3).

[5] Lee K, Chong Y, Shin HB, et al. Modified Hodge and EDTA-disk synergy tests to screen metallo-betalactamase-producing strains of pseudomonas and Acinetobacter species. Clin Microbiol Infect 2001;7(2):88-91.

[6] Yong D, Lee K, Yum JH, et al. Imipenem-EDTA disk method for differentiation of metallo- $\beta$-lactamaseproducing clinical isolates of pseudomonas spp and Acinetobacter spp. J Clin Microbiol 2002;40(10):3798801.

[7] Lee K, Lim YS, Yong D, et al. Evaluation of the Hodge test and the imipenem-EDTA double disk synergy test for differentiating metallo- $\beta$-lactamase-producing isolates of Pseudomonas spp and Acinetobacter spp. J Clin Microbiol 2003;41(10):4623-9.

[8] Agrawal G, Lodhi RB, Kamalakar UP, et al. Study of metallo-beta-lactamase production in clinical isolates of pseudomonas aeruginosa. Indian Journal of Medical Microbiology 2008;26(4):349-51.

[9] Takayama Y, Adachi Y, Nihonyanagi S, et al. Modified Hodge test using Mueller-Hinton agar supplemented with cloxacillin improves screening for carbapenemase-producing clinical isolates of Enterobacteriaceae. Journal of Medical Microbiology 2015;64(7):774-7.

[10] Behera B, Mathur P, Das A, et al. An evaluation of four different phenotypic techniques for detection of metallo-beta-lactamase producing pseudomonas aeruginosa. Indian Journal of Medical Microbiology 2008;26(3):233-7.

[11] Bora A, Ahmad GU, Hazarika NK, et al. Incidence of blaNDM-1 gene in Escherichia coli isolates at a tertiary care referral hospital in Northeast India. Indian Journal of Medical Microbiology 2013;31(3):250-6.

[12] Islam MA, Nabi A, Rahman M, et al. Prevalence of a faecal carriage of NDM-1 producing bacteria among patients with diarrhoea in Bangladesh. Journal of Medical Microbiology 2014;63(Pt 4):620-2. 